\title{
EXPERIMENTAL AND THEORETICAL INVESTIGATION OF SYNERGY BETWEEN ION BERNSTEIN AND LOWER HYBRID WAVES IN PBX-M
}

\author{
F. Paoletti ${ }^{1}$, A. Cardinali2 , S. Bernabei ${ }^{3}$, A. Post-Zwicker ${ }^{3}$, W. Tighe 3 , S. Von Goeler ${ }^{3}$ \\ ${ }^{1}$ Columbia University, Department of Applied Physics, 500 West 120th Street, New \\ York, NY 10027 \\ ${ }^{2}$ Associazione Euratom-ENEA sulla Fusione, Centro Ricerche Energia Frascati, C.P. \\ 65, 00044 Frascati, Rome, Italy \\ 3Princeton University, Plasma Physics Laboratory, Forrestal Campus, P.O. Box 451, \\ Princeton, NJ 08543
}

\begin{abstract}
The synergistic behavior of lower hybrid and ion Bernstein waves on the Princeton Beta Experiment-Modified tokamak [Phys. Fluids B 2, 1271 (1990)] is experimentally studied using a 2-D hard X-ray camera. The hard X-ray bremsstrahlung emission from suprathermal electrons, generated with lower hybrid current drive, is enhanced during ion Bernstein wave power injection. This enhancement is observed in limited regions of space suggesting the formation of localized current channels. The effects on plasma electrons during combined application of these two types of waves are theoretically investigated using a quasilinear model. The numerical code simultaneously solves the 3-D $(R, Z, \Phi)$ toroidal wave equation for the electric field (in the WKBJ approximation) and the Fokker-Planck equation for the distribution function in two dimensions $\left(\mathrm{v}_{\|}, \mathrm{v}_{\perp}\right)$ with an added quasilinear diffusion coefficient. The radial profile of the non-inductively generated current density, the transmitted power traces and the total power damping curve are calculated. The beneficial effects of a combined utilization of ion Bernstein and lower hybrid waves on the current drive are emphasized. The numerical results are compared with the experimental observations.
\end{abstract}




\section{INTRODUCTION}

Lower hybrid wave (LHW) and ion Bernstein wave (IBW) power was simultaneously injected in the Princeton Beta Experiment-Modified (PBX-M) ${ }^{1}$ tokamak plasma to study the possibility of increasing the non-inductive current drive efficiency via a synergistic effect. IBWs are efficient for heating the bulk ions of the plasma at a given harmonic resonance. However, as was previously shown 2,3,4,5, IBWs can also interact directly with the electron population. This is due to the strong increase of the wave's parallel refraction index $\left(n_{\|}-\right.$parallel to the local magnetic field) along the ray trajectory in the WKBJ approximation.

As shown in this paper, IBWs alone are unable to produce a net current in the plasma due to the fact that the launched $n_{\|}$spectrum is symmetric. Nevertheless, when IBWs are used in conjunction with LHWs, a synergistic effect is possible.

Previous studies showed that the IBW $n_{\|}$is continuously changing from positive to negative values along the ray trajectory $3,4,5$. In the spatial regions near the $n_{\|}$maximum values, IBW can induce a quasilinear modification of the distribution function. Some thermal electrons, interacting with the IBW's electric field, are extracted from the bulk and the resulting distribution function is broadened in the velocity space.

When this modification takes place in the same direction of the lower hybrid current drive (LHCD), it contributes to the filling of the LHW spectral gap at very low parallel velocities (high values of $n_{\|}$). Therefore, LHWs do not need to form a tail through upshift. $\mathrm{n}_{\|}$. They can damp at an outer radius generating a current which would be otherwise impossible at that given location. This is the most efficient synergistic interaction found between these two types of waves. 
Another possible synergistic effect between LHWs and IBWs involves the IBWs sustaining part of the non-inductive current while dissipating its energy in the direction of the parallel velocity where the electron tail, formed by $\mathrm{LHCD}^{5}$, already exists. Our calculations, based on the specific experimental case we modeled, show that this effect is much smaller than the one we just described.

The main diagnostic used to detect variations of the electron distribution function is a 2-D Hard X-Ray (HXR) camera6,7. The camera measures the bremsstrahlung emission from fast electrons on the tail of the distribution function. Experimentally, a strong increase in the HXR emission is observed for combined LHW+IBW operation with respect to the LHW alone ${ }^{8,9}$. Careful analysis of the HXR data shows that this variation is not uniform along the plasma radius: localized incremental HXR emission is observed in regions located off-axis.

In our numerical model5,10, we consider the interaction between LHW and IBW coupling a 3-D $(R, Z, \Phi)$ toroidal IBW+LHW ray-tracing algorithm with a 2-D ( $\mathrm{v}_{\|}$, $\mathrm{v}_{\perp}$ ) relativistic Fokker-Plank code ${ }^{11,12}$. The quasilinear distribution function is calculated as a result of the presence of both waves on each magnetic surface. This distribution function is used to calculate the transmitted power, the damping rate, and the non-inductive current density profile.

The paper is organized as follows: In section II, the experimental results from the PBX-M experiment are presented. In section III, the model used in this analysis is described. Section IV contains the numerical results of the model and the comparison with the experimental findings. Conclusions are drawn in section V. 


\section{EXPERIMENTAL RESULTS}

One of the main goals of the LHCD experiment on PBX-M was to modify the current density profile in order to produce a more stable plasma discharge ${ }^{13,14,15}$. Combined application of LHW and IBW power can improve the ability of localizing the current drive via a synergistic interaction between these two types of waves ${ }^{16}$.

The LHW+IBW experiment was performed in circular plasmas, where the LHCD is typically concentrated in the center of the plasma. The aim of this experiment was to identify a possible way to localize part of the non-inductive additional current off-axis. As will be shown, this was possible by combining LHW and IBW power.

The HXR emission is the main diagnostic tool which provides information on the spatial and velocity distribution of the electrons accelerated via Landau damping by LHW. The HXR diagnostic consists of a pinhole camera which provides a complete tangential view of the PBX-M plasma6,7. The 2-D HXR images are integrated over the plasma volume which is covered by the angle of view, as shown in Fig. 1. To obtain HXR emissivity profiles, an inversion of the images is performed. A detailed description of the mathematical and numerical method used for this inversion process has been previously published $7,17,18$.

Our investigation is based on profiles obtained taking a horizontal crosssection of the 2-D image along the mid-plane through the plasma center. In the presence of localized fast electron current channels, the HXR emission should be enhanced in the region where the current channel is generated. In this study, we are detecting the HXR emission from the most external current channel. To do so, we concentrate our analysis on the right hand side (RHS) portion of the volumeintegrated horizontal profile. The HXR emission from electrons moving in the more 
external current channel is not affected by a line integration of the emission from other electrons traveling in more internal current channels (see Fig. 1; the concentric toroidal rings represent a schematic of possible multiple current channels). To detect the HXR emission due to electrons from more internal current channels we would have to perform the mentioned image inversion which introduces several numerical uncertainties limiting the radial spatial resolution ${ }^{19}$.

Furthermore, IBW is known to cause an influx of impurities, which results in a gradual peaking of the total radiated power profile 20 . Since the main effect to be studied is the increase of HXR emission, it is important to distinguish the increase due to accelerated electrons via Landau damping from the increase caused by interactions with impurities. Previous studies showed that there is no accumulation of impurities in any off-axis region 20 , only in the center. Therefore, an off-axis increase of the HXR emission has to be ascribed to the damping of IBW power. In addition, in order to clearly discriminate between impurity effects and IBW damping, the analysis is performed in discharges with limited IBW power, since this limits the influx of impurities.

The parameters of a typical target plasma used in the LHW+IBW experiment on PBX-M are given in Tab. 1. The characteristics of the radio frequency (RF) systems are summarized in Tab. 2. The principal quantity which best describes the effect of IBW during LHCD, is the variation of HXR emission:

$$
\Delta_{H X}(t) \equiv I_{H X}\left(t_{L H}+I_{B W}\right)-I_{H X}\left(t_{L H}\right)
$$

where: $I_{H X}\left(t_{L H}\right)$ is the horizontal HXR intensity at a time frame just before the onset of IBW (i.e., when only $\mathrm{LH}$ is present) and $\mathrm{I}_{\mathrm{HX}}\left(\mathrm{t}_{\mathrm{LH}+\mathrm{IBW}}\right)$ is the horizontal HXR intensity at a time when both types of waves are present in the plasma. In this experiment, the 
data acquisition was programmed to have one HXR image every 5 ms. Each image is referred to as a "time frame".

The radial profiles of $\mathrm{I}_{\mathrm{HX}}(\mathrm{t})$ at different time frames for the same plasma discharge of Tab. 1, are shown in Fig. 2. The frame at $t_{L H W}=400 \mathrm{~ms}$ is considered the LHW-only reference, since it is at this time that IBW is applied. The continuous line in Fig. 2 shows the shape of the vertical HXR intensity at $t_{L H W}$.

During IBW power injection, as discussed earlier, two different effects are observed: a distortion of the HXR emission at a well-defined off-axis location and a global increase primarily concentrated in the central region. The growing phase of this distortion is shown in Fig. 2 while the decay phase, when IBW has been turned off, is shown in Fig. 3. The arrows indicate the evolution of the profiles $\mathrm{I}_{\mathrm{HX}}(\mathrm{t})$ in time.

Fig. 4 and Fig. 5 show in greater detail the evolution of the profile by plotting the incremental $\mathrm{HXR}$ emission $\Delta_{H X}(\mathrm{t})$ at several time frames.

Fig. 4 shows the radial profiles of the variable $\Delta_{H X}(t)$ for the initial four time frames following the onset of IBW injection. At $\mathrm{t}=405 \mathrm{~ms}$ (time-frame \# 23 in Fig. 4) an increase of the HXR emission is observed, which starts to build up from the center. At $\mathrm{t}=410 \mathrm{~ms}$ (time-frame \# 24 in Fig. 4), this centrally-peaked distortion extends up to $R \cong 182 \mathrm{~cm}$. At $t=415 \mathrm{~ms}$ (time-frame \# 25 in Fig. 4), a distinct peak begins to emerge around $R \cong 180 \mathrm{~cm}$. At $\mathrm{t}=415 \mathrm{~ms}$ (time-frame \# 26 in Fig. 4), the peak is very well-formed and represents the major part of the distortion in the HXR emission. Clearly, the interaction between LHW and IBW starts in a region located off- axis and the increase of $\mathrm{I}_{\mathrm{HX}}(\mathrm{t})$ indicates an increase of the fast electron population.

From Fig. 5, it is possible to see that the peak in $\Delta_{\mathrm{HX}}(\mathrm{t})$ moves further out from its original position, while its the amplitude increases. The same effect is visible on 
$\mathrm{I}_{\mathrm{HX}}(\mathrm{t})$ by comparing time-frame \# 26 with time-frame \# 32 of Fig. 2 . The HXR profile broadens and increases.

After IBW is turned off at $t=450 \mathrm{~ms}$ (time-frame \# 32 in Fig. 5), the off-axis peak of $\Delta_{\mathrm{HX}}(\mathrm{t})$ continues to rise for about $10-15 \mathrm{~ms}$. This is consistent with the characteristic fast electron thermalization time scale on PBX-M. The LHW continues damping on a fast electron tail which is relaxing after the IBW is turned off. After this, the $\Delta_{H X}(t)$ profile continues to evolve, the off-axis peak is lost (time-frames \# 34, 35 in Fig. 5) and most of the distortion in the HXR emission appears to concentrate in the center (time-frame \# 39 in Fig. 5). The full relaxation of the HXR emission profile to its original shape and value takes place on a longer time scale, as clearly seen in Fig. 3 for $\mathrm{t}=600 \mathrm{~ms}$ (see also time-frame \# 62 in Fig. 5 with $\Delta_{H X}(\mathrm{t})$ approaching zero). This observation can be correlated with the radial diffusion and loss of the impurities previously accumulated in the center.

Fig. 6 shows the time dependent behavior of the central emitting Fe XVI (335 $\AA$ ) emission line normalized to the electron density as detected by the SPRED (Survey Poor Resolution Extended Domain) spectrometer21. The decrease in the intensity of the line emission, after the IBW power has been turned off, is consistent with a radial diffusion of the impurities from the center toward the edge 20 . On a same order time scale, we observe a full relaxation of the HXR emission profile (see timeframe \# 39 and \# 62 in Fig. 5).

In conclusion, we can say that the observed off-axis distortion in the HXR horizontal profiles cannot be ascribed to fast electron bremsstrahlung emission from interaction with accumulated impurities since these are concentrated in the central regions. We consider this as the experimental proof of the existence of a localized current channel generated by the synergy between LHW and IBW in PBX-M. 


\section{THEORETICAL INTERPRETATION AND MODELING}

A numerical code $5,22,23$ has been used to simulate the IBW-LHW synergy and to model the behavior of both waves in the PBX-M plasmas. The code includes the dynamical evolution of the trajectories of both waves and the 2-D (in the velocity space: $v_{\|}, v_{\perp}$ ) solution of the Fokker-Planck equation ${ }^{11,12}$ with an added quasilinear term.

Among the numerical outputs of the code are the radial profiles of the power absorbed, the generated additional current density, and the mutual damping of both waves on the quasilinear modified distribution function. The distortion of the distribution function is the effect of the interaction between the electric fields of the propagating waves with the plasma electron population.

In this simulation, attention has been mainly focused on the effect that the IBW produces on a discharge to which LHW have been already applied. An extensive analysis of the lower hybrid experiments on PBX-M, and a comparison between the results of the simulation and the experimental data, can be found in Ref. 19. In the following section, we outline the method followed in the simulation code to obtain absorbed power and current generated profiles.

The propagation characteristics of both waves are included in the code in the analytical form which has been published in Refs. $(3,24)$. In these papers, the raytracing and power transport equations of the lower hybrid and ion Bernstein waves (in the cold electrostatic approximation) were analytically solved. The solution is found for a generic 2-D toroidal geometry in the limit of small inverse aspect ratio (quasi-cylindrical geometry).

The result of this analytical integration relies on the determination of the evolution of the parallel wavenumber $n_{\|}$and the WKBJ amplitude of the electric field 
along the trajectory. This allows the calculation of the quasilinear diffusion coefficient and the interval, in the parallel velocity space, where the wave electric field is localized.

After performing an average over the magnetic surface and normalizing the result to the collisional diffusion coefficient, the quasilinear diffusion coefficient can be written as:

$$
<D_{Q L}\left(v_{\|}, \rho\right)>=\Sigma_{\psi}^{-1} \int_{\Delta \Sigma_{\psi}} d \Sigma_{\psi} \frac{\pi}{v_{v}^{e e} v_{\text {the }}^{2}}\left(\frac{e}{m_{e}}\right)^{2} \frac{1}{L_{\infty}} \int_{-\infty}^{+\infty}\left|E_{\|}\left(n_{\|}, \theta, \rho\right)\right|^{2} \delta\left(\omega-k_{\|} v_{\|}\right) d n_{\|}
$$

where $\left|E_{\|}\left(n_{\|}, \theta, \rho\right)\right|^{2}$ is the squared amplitude of the Fourier analyzed parallel electric field; vee is the electron-electron collision frequency; $v_{\text {the }}$ is the electron thermal velocity; $\rho$ and $\theta$ are the radial and the poloidal angle coordinates; $\Sigma_{\psi}$ is the total area of the magnetic surface under consideration; and $\Delta \Sigma_{\psi}$ is the image of the antenna's area over the magnetic surface.

From the Poynting theorem, we obtain the expression of the squared field amplitude in terms of the antenna power spectrum:

$$
\left|E_{\| \mid}\left(n_{\|}, \theta, \rho\right)\right|^{2}=\frac{P\left(n_{\|}, \theta, \rho\right)}{R \Delta \phi \rho \Delta \theta(\rho) \mathbf{v}_{g} \cdot n_{\psi}}
$$

where $R=R_{0}\left(1+\rho / R_{0}\right)$ is the major radius of the torus; $\phi$ is the toroidal angle; $\mathbf{v}_{g}$ is the time-normalized group velocity $\mathbf{v}_{\mathrm{g}}=\partial \mathrm{H} / \partial \mathbf{k}$ (with $\mathrm{H}$ the dispersion relation); and $\mathbf{n}_{\psi}$ is the unity vector perpendicular to the magnetic surface. In deriving Eq. (2), we have made the hypotesis that the electric field weakly depends on the poloidal angle $\theta$ as 
well as the dispersion relation. In other words, we have neglected the terms of order $\varepsilon$ (inverse aspect ratio) in the calculation of the surface integral of the Poynting equation, and we have expressed the area as $\Delta \Sigma_{\psi}=R \Delta \phi \rho \Delta \theta(\rho)$. It is important to note that $\Delta \theta$ in Eq. (2) is a function of $\rho$, thereby simulating the effect of the ray trajectories focusing.

Using Eq. (2) in Eq. (1) and performing the integral, we obtain the following quasilinear diffusion coefficient:

$$
\begin{aligned}
& \left.<\mathrm{D}_{\mathrm{QL}}\left(\mathrm{v}_{\|}, \rho\right)>=\Sigma_{\psi}^{-1} \int \mathrm{d} \Sigma \frac{16 \pi^{2}\left(\mathrm{e} / \mathrm{m}_{\mathrm{e}}\right)^{2} \mathrm{P}_{\mathrm{cgs}}}{v^{\mathrm{ee}} \mathrm{v}_{\text {the }}^{2}} \omega \frac{\mathrm{P}_{\| \mathrm{NOR}}\left(\mathrm{n}_{\|}, \theta\right) \exp \left(-2 \int_{0}^{\mathrm{t}} \Gamma \mathrm{dt}\right) \delta\left(\frac{\mathrm{c}}{\mathrm{v}_{\|}}-\mathrm{n}_{\|}\right)}{\left(1+\alpha_{\mathrm{pol}}^{2}\right)\left(\partial \mathrm{H} / \partial \mathrm{n}_{\psi}\right) \Delta \Sigma_{\psi}}\right] \\
& \Delta \Sigma_{\psi}
\end{aligned}
$$

with

$$
\Gamma=\frac{\mathbf{k} \cdot \varepsilon^{\mathrm{A}} \cdot \mathbf{k}}{|\mathrm{k}|^{2}(\partial \mathrm{H} / \partial \omega)}=\frac{2 \pi^{2}}{\partial \mathrm{H} / \partial \omega} \frac{\omega_{\mathrm{pe}}^{2}}{\omega^{2}} \frac{\mathrm{k}_{\|}}{\left|\mathrm{k}_{\|}\right|}\left[\mathrm{k}_{\|} \omega \mathrm{v}_{\mathrm{the}} \mathrm{x}_{0 \mathrm{e}}\right] \int_{0}^{\infty} \mathrm{v}_{\perp} \mathrm{dv} \mathrm{v}_{\perp} \frac{\partial \mathrm{F}_{0 \mathrm{e}}}{\partial \mathrm{v}_{\|}}
$$

which is the quasilinear damping rate. In Eqs. (2) and (3), $v_{\|}$is the resonant parallel velocity $\left(v_{\|}=c / n_{\|}\right) ; \alpha_{p o l}=E_{\perp} / E_{\|}=k_{\perp} / k_{\|}$is the polarization factor which can be deduced from the dispersion relation; and $\left(\partial \mathrm{H} / \partial \mathrm{n}_{\psi}\right)$ is the time-normalized group velocity perpendicular to the magnetic surface $\left(\left(\partial \mathrm{H} / \partial \mathrm{n}_{\psi}\right)=\mathrm{v}_{\text {group }}(\partial \mathrm{H} / \partial \omega)\right)$. In Eq. (2), $\mathrm{Pcgs}$ is the total launched power in cgs units and $\mathrm{P}_{\mathrm{NOR}}$ is the normalized power spectrum. For the sake of simplicity, we can assume $P_{N O R}=\frac{1}{\pi^{1 / 2} \Delta \theta} \exp \left[-\left(\frac{\left(n_{\|}-n_{\| c}\right)}{\delta n_{\|}}\right)^{2}\right]$ with a uniformly distribution on the poloidal extension of the antenna $\Delta \theta$. 
In Eq. (3), we can approximate the integrand, retaining only the zeroth order terms in the aspect ratio which are independent of $\theta$ and perform the integral to obtain the following relation:

$$
<\mathrm{D}_{\mathrm{QL}}\left(\mathrm{v}_{\|}, \rho\right)>=\frac{16 \pi^{2}\left(\mathrm{e} / \mathrm{m}_{\mathrm{e}}\right)^{2} \mathrm{P}_{\mathrm{cgs}}}{\Sigma_{\psi} \nu^{\mathrm{ee}} \mathrm{v}_{\text {the }}^{2} \omega\left|\mathrm{v}_{\|}\right|} \cdot\left[\frac{\mathrm{P}_{\mathrm{NOR}}\left(\mathrm{n}_{\|}\right) \exp \left(-2 \int_{0}^{\mathrm{t}} \Gamma \mathrm{dt}\right) \delta\left(\frac{\mathrm{c}}{\mathrm{v}_{\|}}-\mathrm{n}_{\|}\right)}{\left(1+\alpha_{\text {pol }}^{2}\right)\left(\partial \mathrm{H} / \partial \mathrm{n}_{\psi}\right)}\right]
$$

This is consistent with the previous approximation taken when integrating the Poynting equation. In Eq. (4), $\underline{\varepsilon}^{\mathrm{A}}$ is the antihermitian part of the dielectric tensor which accounts for the quasilinear absorption. $\underline{\varepsilon}^{A}$, in fact, depends on the derivative of the distribution function $\left(\mathrm{F}_{0 \mathrm{e}}\right)$ with respect to the resonant parallel velocity (see the r.h.s. of Eq. 4). The knowledge of the electron distribution function arises from the numerical integration of the Fokker-Planck equation.

The quasilinear diffusion coefficient of Eq. (5) is the general one for electrostatic waves. In our particular problem, we must calculate the quasilinear diffusion coefficient $<D_{Q L}\left(v_{\|}, \rho\right)>$ for lower hybrid and lon Bernstein waves. To do this, we need to recall the expressions of the LHW and IBW dispersion relations. In particular, for LHWs, we have:

$$
H^{\mathrm{lhw}}=1+\left(-\frac{\omega_{\mathrm{pi}}^{2}}{\Omega_{\mathrm{ci}}^{2}}+\frac{\omega_{\mathrm{pe}}^{2}}{\Omega_{\mathrm{ce}}^{2}}\right) \frac{n_{\perp}^{2}}{n^{2}}-\left(\omega_{\mathrm{pi}}^{2}+\omega_{\mathrm{pe}}^{2}\right) \frac{\mathrm{n}_{\|}^{2}}{n^{2}}
$$

while for IBWs ${ }^{3}$ :

$$
H^{\text {ibw }}=1-\left(\frac{m_{i}}{m_{e}}\right) \frac{k_{\|}^{2} \rho_{i}^{2}}{2 N^{2}}-\frac{\omega}{\pi^{1 / 2} k_{\perp} \rho_{i}\left(\omega-N \Omega_{c i}\right)}
$$


Making use of the dispersion relations Eqs. (6) and (7), it is possible to calculate the time-normalized group velocity, and hence the quasilinear diffusion coefficient.

In the case of LHWs we find that $\left\langle\mathrm{D}_{\mathrm{QL}}\left(\mathrm{v}_{\|}, \rho\right)>\right.$ is proportional to the square of the parallel wavenumber and it follows its evolution along the ray trajectory. Elsewhere 24 , we have found that the parallel wavenumber for lower hybrid waves evolves along the ray trajectory as:

$$
<n_{\|}>=n_{\| 0}+\left(<n_{\| 0}>-n_{\| 0}\right) \frac{q_{a}}{q(x)}-\frac{\varepsilon^{2} n_{\| 0}}{2 q(x)}\left[q_{a}-x^{2} q(x)\right]
$$

where the brackets " $<\ldots>$ " indicate poloidal angle averaged quantities; $\mathrm{q}(\mathrm{x})$ is the safety factor profile; $<n>$ is a constant (see Ref. 24); and $\varepsilon$ is the inverse aspect ratio.

In the case of IBWs, the quasilinear diffusion coefficient is proportional to the cubic power of the parallel wavenumber, which evolves as 5 :

$$
\mathrm{n}_{\|}(\mathrm{x})=\Lambda(\mathrm{x})\left[-\delta_{\mathrm{T}}^{-1 / 2}\left(\frac{\mathrm{m}_{\mathrm{e}}}{\mathrm{m}_{\mathrm{i}}}\right)^{1 / 2} \frac{N \theta_{0}}{\rho_{\mathrm{i}}} \sin (v(\mathrm{x}))+\mathrm{n}_{\| 0} \cos (v(\mathrm{x}))\right]
$$

where: $\delta_{\mathrm{T}}^{-1 / 2}=\mathrm{T}_{\mathrm{i}}(\mathrm{a}) / \mathrm{T}_{\mathrm{i}}(0)$ with $\mathrm{T}_{\mathrm{i}}(0)$ and $\mathrm{T}_{\mathrm{i}}(\mathrm{a})$ being the central and edge ion temperatures; $T_{i}(x)$ is the ion temperature profile $\theta_{0}$ is the poloidal launching angle; $\rho_{\mathrm{i}}$ is the ion gyroradius normalized to $c / \omega ; N$ is the harmonic number resonance; $n_{\| 0}$ is the launched wavenumber; and $\mathrm{x}$ is the distance from the resonance location which, for the sake of simplicity, has been assumed to be in the plasma center. 
The oscillation frequency $v(\mathrm{x})$ of Eq. 9 is defined as ${ }^{5}$ :

$$
v(x)=\sqrt{\frac{m_{i}}{m_{e} \pi N^{2} q_{0}^{2}}} \ln \left(\frac{x \sqrt{1+x_{c}^{2}}}{\sqrt{x^{2}+x_{c}^{2}}}\right) ;
$$

with $x_{c}=\frac{1}{\sqrt{q_{a} / q_{0}-1}}$ where $q_{a}$ and $q_{0}$ are the edge and central values of the safety factor respectively.

The overall amplitude $\Lambda(x)$ of Eq. 9 is defined as 5 :

$$
\Lambda(x)=\left(\frac{T_{i}(a)}{T_{i}(x)}\right)^{1 / 4}\left(\frac{q_{a}}{q(x)}\right)^{1 / 2}
$$

where $q(x)$ is the safety factor profile.

The IBW quasilinear diffusion coefficient follows the oscillation of the parallel wavenumber. This signifies that the wave interacts strongly with the electrons in the bulk of the distribution only in points where the $n_{\|}$oscillation reaches a maximum (low parallel velocities).

All the quantities defined above can be calculated in the ray-tracing routine and used as an input for the Fokker-Planck calculation. The quasilinear diffusion coefficient and the spread of the waves in the velocity space are needed as input to the Fokker-Planck routine.

After running the Fokker-Planck routine, the distribution function is obtained and it can be used to calculate the quasilinear damping on that magnetic surface as well as the absorbed power and current generated densities. The damped power can be used to calculate the new quasilinear diffusion coefficient on the next surface where the procedure outlined before is repeated. Between the two adjacent 
magnetic surfaces $\left(\psi_{1}=\psi_{0}+\Delta \psi\right.$ and $\left.\psi_{2}=\psi_{1}+\Delta \psi\right)$ the damped power is considered constant.

Proceeding in this way, we can follow the ray's trajectories. At the same time, we can have a self-consistent calculation of the damping, power absorption and current density profiles.

In next section, the results of the numerical analysis applied to the PBX-M tokamak are presented and compared to the experimental data. 


\section{NUMERICAL RESULTS AND COMPARISON WITH THE EXPERIMENT}

Using a numerical MHD equilibrium ${ }^{25}$ with the parameters relative to Tab. 1, a model for a target discharge is obtained. For this plasma, as described in the previous section, the LHW and IBW WKBJ ray-tracing equations are integrated. The quasilinear diffusion coefficients are calculated at each step of integration using the values of the wave electric field components. The parameters of the applied waves are shown in Tab. 2. Different numerical setups were prepared to test the contribution on one type of wave versus the other in the interaction process that determines the synergy.

In the first setup, the Fokker-Planck equation is solved along both waves ray trajectories, assuming $\mathrm{D}_{\mathrm{QL}}(\mathrm{IBW})=0$ and $\mathrm{D}_{\mathrm{QL}}(\mathrm{LHW}) \neq 0$. This case is compared with the general setup where both waves quasilinear diffusion coefficients are allowed to be non zero. This is to test the ability of IBW in modifying the electron distribution function and the influence that this modification has on the damping of LHW. Figs. 7 and 8 respectively represent and the IBW the LHW transmitted power rate radial profiles.

To analyze the result shown in Fig. 7 it is necessary to recall, as stated in the previous section, that an analytical solution of the IBW set of ray-tracing equations was obtained in previous studies $3,4,5$. Following that formalism, it is found that the IBW parallel wave number oscillates along the trajectory 4,5 with a frequency that increases as the ray approaches the plasma center (see Eq. (10) in section III). Since the IBW electric field is proportional to $\left|\mathrm{n}_{\|}(\mathrm{x})\right|^{3}$, the resulting diffusion coefficient is also oscillating. 
Due to this oscillatory behavior, quasilinear modifications of the distribution function are localized in space. A detailed discussion of this phenomenology can be found in Ref. 5. The IBW transmitted power rate profiles also follow the oscillation of $\mathrm{n}_{\|}(\mathrm{x})$. The spatial locations of the IBW $\mathrm{n}_{\|}(\mathrm{x})$ maxima can be varied by changing plasma parameters such as $q_{a}$ and $q_{0}$ or the wave ion harmonic number $N$ (see Eq. (10) in section III).

In the case shown in Fig. 7, the IBW damping rate is reduced when the quasilinear distortion is turned on ( $\mathrm{D}_{\mathrm{QL}}(\mathrm{IBW}) \neq 0$, filled circles) when compared with the case where there is no quasilinear modification of the electron distribution function due to IBW $\left(D_{\mathrm{QL}}(\mathrm{IBW})=0\right.$, crosses $)$.

This result can be explained by noticing that the modification of the distribution function takes place in the regions around the maxima of $n_{\|}(x)$. The IBW power deposition is also strong in these regions. This implies that IBW damps its power preferentially on low $v_{\|}$electrons (high $n_{\|}$) from the bulk of the distribution.

The quasilinear modification of the distribution function due to IBW injection results in a broadening of the electron distribution shape along the $v_{\|}$axis since the electrons that are interacting with the electric field of the wave are accelerated to higher parallel velocities 5 . The Landau damping of IBW waves on such modified distribution function results to be weaker when compared to the case with $D_{Q L}($ IBW $)=0$ because the derivative term $\frac{\partial F_{0 e}}{\partial v_{\|}}$is smaller in the regions where most of the wave-electrons interaction takes place (see Eq. (4) in the previous section).

The IBW quasilinear modification of the distribution function also affects the LHW damping but in the exactly opposite way. In fact, (see. Fig. 8), the LHW transmitted power rate drops considerably in the region around $x \approx 0.6$ compared with the case where $D_{Q L}(I B W)=0$. 
This is the effect at the core of the synergistic interaction between IBW and LHW, as described in section II. It is possible now to compare the numerical results with the experimental observations shown there.

The rising of an off-axis peak in the radial profile of $\Delta_{\mathrm{HX}}(\mathrm{t})$ can be correlated with the damping of LHW on the tail of a quasilinearly modified distribution function due to IBW. As stated before, the IBW is creating a symmetric tail in the distribution function in the regions around its $n_{\|}(x)$ maxima. The incoming $\mathrm{LH}$ waves can damp on "first-pass" on this tail creating localized current channels.

The shift of $\Delta_{\mathrm{HX}}(\mathrm{t})$ peak towards more peripheral regions observed in Fig. 5, can be related to a radial diffusion of the fast electron population ${ }^{17}$ which extends the suprathermal electron tail further out in radius. In turn, this extends the potential LHW "first-pass" damping on the tail of the distribution further out creating a looptype effect.

Another experimental measurement that is supporting the proposed interpretation is the behavior of the $\Delta_{\mathrm{HX}}(\mathrm{t})$ profile (shown in Fig. 5) during the time interval between $\mathrm{t}=450 \mathrm{~ms}$ and $\mathrm{t}=465 \mathrm{~ms}$. The fact that the $\Delta_{H X}(\mathrm{t})$ off-axis peak continues to rise even after the IBW power source has been turned off supports the hypothesis that during this period of time LHWs alone are damping off-axis on a tail that is progressively disappearing.

When the $I_{H X}$ profile starts relaxing toward its original shape (see Fig. 3 in the time window from $t=465 \mathrm{~ms}$ to $t=600 \mathrm{~ms}$ ) the off-axis distortion in the HXR emission disappears. Here, in fact, LHWs no longer find enough fast electrons in the off-axis regions and they are required to travel more toward the center to deposit their power. As shown in section II, the relaxation of the $\mathrm{I}_{\mathrm{HX}}$ profile is consistent with the outward diffusion of the plasma impurities accumulated in the center. 
The remaining two numerical calculations are intended to check the importance of the other synergistic interaction between IBW and LHW, mentioned in section I. That is the damping of IBW on a quasilinear distribution function modified by the presence of LHCD [i.e. $D_{Q L}(I B W)=0$ and $D_{Q L}(L H W) \neq 0$ ]. versus the damping on a Maxwellian distribution function [i.e. $\mathrm{D}_{\mathrm{QL}}(\mathrm{IBW})=0$ and $\mathrm{D}_{\mathrm{QL}}(\mathrm{LHW})=0$ ] .

Fig. 9 shows the IBW transmitted power for these two cases. The difference is almost inappreciable. From this, it is concluded that most of the IBW power is damped on the bulk of the distribution and the interaction of low $n_{\|}$IBW waves with the fast electrons generated by LHCD is very weak in the specific experimental case modeled.

The LHW absorbed power radial profiles are shown in Fig. 10. For the case where $\mathrm{D}_{\mathrm{QL}}(\mathrm{IBW}) \neq 0$ and $\mathrm{D}_{\mathrm{QL}}(\mathrm{LHW}) \neq 0$ (filled circles) a series of peaks are seen around the locations where $n_{\|}(I B W)$ reaches its maxima. The other curve (crosses) represents the LHW absorbed power in the case where there is no quasilinear distortion of the distribution function due to IBW (i.e. $D_{Q L}(I B W)=0$ and $\left.\mathrm{D}_{\mathrm{QL}}(\mathrm{LHW}) \neq 0\right)$. From this comparison, it is possible to conclude that the additional LHW power deposited in the localized off-axis regions (i.e. the peaks in Fig. 10) is enhancing the current drive in these regions.

The total non inductively driven current density profile is obtained from the code as the first moment of the quasilinearly modified distribution function. Fig. 11 shows the calculated non-inductive current density profile for the general case $\left(D_{\mathrm{QL}}(\mathrm{IBW}) \neq 0\right.$ and $\mathrm{D}_{\mathrm{QL}}(\mathrm{LHW}) \neq 0$ ) compared with the case in which $\mathrm{D}_{\mathrm{QL}}(\mathrm{IBW})=0$. In the latter case, only LHWs are driving additional non inductive current while there is no appreciable contribution from IBW (see discussion of Fig. 9). In the former case, the synergistic interaction between the two types of waves generates localized 
current channels in different radial locations corresponding with the spatial regions where the $n_{\|}($IBW) reaches its maxima.

As described in section II, it was possible to experimentally detect only the first current channel. For the specific case of the plasma discharge of Tab. 1, the IBW $\mathrm{n}_{\|}(\mathrm{x})$ reaches its first maximum around $\mathrm{x} \approx 0.56$ (see Fig. 12) which correspond to a radial location of $R=181.9 \mathrm{~cm}$. A "region of strong synergistic interaction" has been defined for the radii where the value of $n_{\|} \geq 0.5 \bullet n_{\|}$(peak). This defines an interval $R_{0.5}=[179.7,184.5] \mathrm{cm}$. The defined "region of strong synergistic interaction" is highlighted in Fig. 12 with a shaded background.

Comparing these results with the experimental findings shown in Figs. 2 to 5 , it is concluded that the location of the peak seen in the HXR images is consistent with the location of the "region of strong synergistic interaction" defined around the first maximum of the IBW $\mathrm{n}_{\|}(\mathrm{x})$ oscillation. 


\section{CONCLUSIONS}

In this paper, a strong experimental confirmation of a synergistic interaction between lower hybrid and ion Bernstein waves on PBX-M is presented. Making use of data obtained with a 2-D HXR camera, a variation in the HXR emission profile located in an off-axis region of the plasma was observed. The variation of the HXR emission due to the influx of impurities, during IBW injection, is primarily concentrated in the central region during combined LHW + IBW injection. Therefore, the observed off-axis distortion of the HXR emission can be ascribed to a synergistic interaction between IBW and LHW with the formation of localized non-inductive current channels. This result is particularly important because it shows that, during central LHCD, part of the non inductive current can be localized off-axis using combined IBW injection.

The theoretical investigation included the use of a toroidal 3-D LHW+IBW raytracing + 2-D Fokker-Planck numerical code. The results can be summarized as follows: due to the IBW $n_{\|}(x)$ oscillatory behavior, the IBW power is deposited in limited regions of space. The quasilinear distortions of the distribution function due to IBW injection are localized in space and take place around the IBW $n_{\|}(x)$ maxima. During central LHCD, a portion of the LHW power can be deposited off-axis with a "first pass" damping on the electron tail sustained by IBW. The result is the generation of localized current channels. The application of the model to a specific experimental condition has proven that the HXR emission enhancement happens at the location where the first maximum of $n_{\|}$for IBW occurs. 


\section{ACKNOWLEDGMENTS}

We acknowledge G. Menard for the help in several numerical setups, and

L.M. Carlucci for her editorial comments. This work was supported by the US Department of Energy contracts: DE-FG02-89ER53297 and DE-AC02-76CH03073. 


\section{REFERENCES}

1 R. Bell, N. Asakura, S. Bernabei, M.S. Chance, P.A. Duperrex, R.J. Fonck, G.M. Gammel, G.J. Greene, R.E. Hatcher, A. Holland, S.C. Jardin, T.W. Jiang, R. Kaita, S.M. Kaye, C.E. Kessel, H.W. Kugel, B. Leblanc, F.M. Levinton, M. Okabayashi, M. Ono, S.F. Paul, E.T. Powell, Y. Qin, D.W. Roberts, N.R. Sauthoff, S. Sesnic, and H. Takahashi, Phys. Fluids B 2, 1271 (1990).

2 M. Ono, Phys. Fluids B 5, 241 (1993).

3 A. Cardinali, and F. Romanelli, Phys. Fluids B 4, 504 (1992).

$4 \quad$ A. Cardinali, Phys. Fluids B 5, 2778 (1993).

5 A. Cardinali, F. Paoletti, S. Bernabei, and M. Ono, Phys. Plasmas 2, 1510 (1995).

6 S. von Goeler, H. Fishman, G. Gettelfinger, D. Ignat, S. Jones, P. Roney, J. Stevens, S. Bernabei, W. Davis, R. Kaita, F. Paoletti, G. Petravich, and F. Rimini, Phys. Plasmas 2, 205 (1995).

7 S. von Goeler, S. Jones, R. Kaita, S. Bernabei, W. Davis, H. Fishman, G. Gettelfinger, D. Ignat, F. Paoletti, G. Petravich, F. Rimini, P. Roney, J. Stevens, and W. Stodiek, Rev. Sci. Instrum. 65, 1621, (1994). 
W. Tighe, R. Bell, S. Bernabei, A. Cardinali, T.K. Chu, S. Jones, R. Kaita, B. Leblanc, M. Ono, F. Paoletti, G. Petravich, H. Takahashi, S. Von Goeler, A. Post Zwicker, and PBX-M group, Bull. Am. Phys. Soc. 38, 2094 (1993).

9 A. Cardinali, S. Bernabei, D. Ignat, D. Lee, M. Ono, F. Paoletti, E. Valeo, and PBX-M group, Bull. Am. Phys. Soc. 38, 2094 (1993).

F. Paoletti, A. Cardinali, M. Shoucri, A Shkarofsky, S. Bernabei, and M. Ono, Proceedings of the $11^{\text {th }}$ Topical Conference on Radio Frequency Power in Plasmas, edited by R. Prater and V.S. Chan, (AIP, Palm Springs, California, 1995), p. 297.

M. Shoucri, and I. Shkarofsky, Comput. Phys. Commun. 78, 199 (1993).

M. Shoucri, and I. Shkarofsky, Comput. Phys. Commun. 82, 287 (1994).

S. Bernabei, R. Bell, M. Chance, T.K. Chu, M. Corneliussen, W. Davis, G. Gettelfinger, T. Gibney, N. Greenough, R. Hatcher, H. Hermann, D. Ignat, S. Jardin, R. Kaita, S. Kaye, C. Kessel, T. Kozub, H. Kugel, L. Lagin, B. LeBlanc, J. Manickam, M. Okabayashi, H. Oliver, M. Ono, S. Paul, S. Preische, P. Roney, N. Sauthoff, S. Schweitzer, S. Sesnic, Y. Sun, H. Takahashi, W. Tighe, E. Valeo, S. Von Goeler, K. Voss, M. Mauel, G. Navratil, R. Cesario, S. Batha, F. Levinton, F. Rimini, N. Asakura, S. Jones, J. Kesner, S. Luckhardt, F. Paoletti, P. Woskow, A Zolfagari, T. Seki, J. Bell, J. Dunlap, A. England, D. Greenwood, J. Harris, G. Henkel, S. Hirshman, R. 
Isler, D. Lee, L. Blush, R. Conn, R. Doerner, Y. Hirooka, R. Lehmer, L. Schmitz, and G. Tynan, Phys. Fluids B, 5, 2562, (1993).

F. Paoletti, D.W. Ignat, J. Kesner, S. Bernabei, R. Kaita, B. LeBlanc, F.M. Levinton, and S.C. Luckhardt, Nucl. Fusion 34, 771, (1994).

J. Kesner, F. Paoletti, S. Bernabei, S. Jones, R. Kaita, C.E. Kessel, F.M. Levinton, S.C. Luckhardt, M. Okabayashi, and S. von Goeler, Nucl. Fusion 34, 619, (1994).

F. Paoletti, A. Cardinali, S. Bernabei, W. Tighe, S. Von Goeler, Bull. Am. Phys. Soc. 42, 2077 (1997).

S.E. Jones, J. Kesner, S. Luckhardt, F. Paoletti, S. von Goeler, S. Bernabei, R. Kaita, G. Giruzzi, and the PBX-M Team, Phys. Plasmas 2, 1548 (1995). R. Bell, Rev. Sci. Instrum. 66, 558, (1995).

S. Bernabei, A. Cardinali, G. Giruzzi, G. T. Hoang, D. Ignat, R. Kaita, M. Okabayashi, F. Paoletti and S. von Goeler, Phys. of Plasmas 4125 (1997).

A. Post-Zwicker, R.C. Isler, W. Tighe, S.F. Paul, M. Ono, B. LeBlanc, R.E. Bell, H.W. Kugel, R. Kaita, Nucl. Fusion 35, 215, (1995). 
F. Paoletti, A. Cardinali, S. Bernabei, R. Kaita, J. Menard, M. Ono, W. Tighe, S. Von Goeler, Bull. Am. Phys. Soc. 41, 1426 (1996). of the $12^{\text {th }}$ Topical Conference on Radio Frequency Power in Plasmas, edited by P.M. Ryan and T. Intrator, (AIP, Savannah, Georgia, 1997), p. 173.

24 A. Cardinali, F. Romanelli, Phys. of Fluids 298101986. A. Cardinali, P. Micozzi, E. Barbato, and F. Romanelli, Phys. Fluids B 5, 2922 (1993). 


\begin{tabular}{|l|c|}
\hline$I_{p}(k A)$ & 120 \\
\hline$B_{0}(T)$ & 1.89 \\
\hline$n_{e}(0) 10^{13}\left(\mathrm{~cm}^{-3}\right)$ & 2.5 \\
\hline$n_{e}(a) 10^{12}\left(\mathrm{~cm}^{-3}\right)$ & 2.5 \\
\hline$T_{e}(0)(\mathrm{keV})$ & 1.1 \\
\hline$T_{e}(\mathrm{a})(\mathrm{eV})$ & 110 \\
\hline$T_{i}(0)(\mathrm{keV})$ & 1.1 \\
\hline$T_{i}(\mathrm{a})(\mathrm{eV})$ & 110 \\
\hline$R_{\mathrm{mag}}(\mathrm{cm})$ & 164 \\
\hline $\mathrm{a}(\mathrm{cm})$ & 32 \\
\hline $\mathrm{q}(0)$ & 0.8 \\
\hline $\mathrm{q}(\mathrm{a})$ & 3.0 \\
\hline
\end{tabular}

Tab. 1

Plasma parameters during LHW+IBW experiments on PBX-M Circular discharge \# 312205 


\begin{tabular}{|l|c|}
\hline \multicolumn{1}{|c|}{ IBW } \\
\hline Frequency $(\mathrm{MHz})$ & 43 \\
\hline $\mathrm{n}_{/ /}$spectrum & $2-16$ \\
\hline $\mathrm{n} / /$ peak & 9 \\
\hline Power $(\mathrm{kW})$ & 50 \\
\hline Pulse Duration $10^{-3}(\mathrm{~s})$ & $400-450$ \\
\hline Loop Antenna Dimensions $(\mathrm{cm})$ & $15 \times 40$ \\
\hline
\end{tabular}

\begin{tabular}{|l|c|}
\hline \multicolumn{1}{|c|}{ LH } \\
\hline Frequency $(\mathrm{GHz})$ & 4.6 \\
\hline $\mathrm{n}_{/ /}$spectrum & $1.9-2.3$ \\
\hline $\mathrm{n} / /$ peak & 2.1 \\
\hline Phasing $\left(^{\circ}\right)$ & 90 \\
\hline Power $(\mathrm{kW})$ & 139 \\
\hline Pulse Duration $10^{-3}(\mathrm{~s})$ & $300-600$ \\
\hline Number of Waveguides & 32 \\
\hline Antenna - Height $(\mathrm{cm})$ & 6 \\
\hline Antenna - Length $(\mathrm{cm})$ & 26 \\
\hline
\end{tabular}

Tab. 2

Waves parameters during LHW+IBW experiments on PBX-M Circular discharge \# 312205 


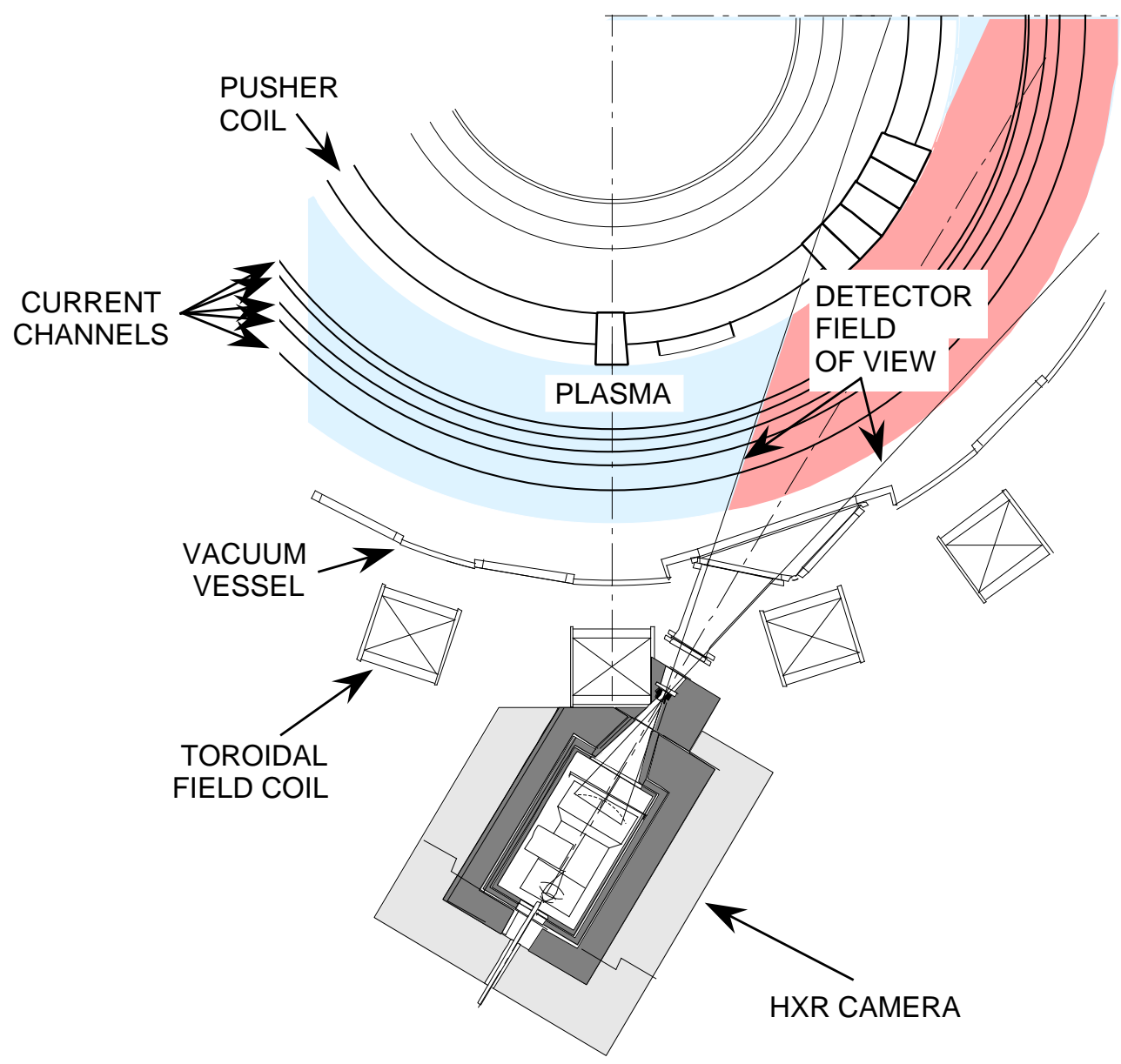

Fig. 1

Schematic of the HXR diagnostic setup on PBX-M. The shaded darker region shows the angle of view of the camera. The toroidally concentric rings represent the region of enhanced HXR emission from electrons traveling in localized current channels 


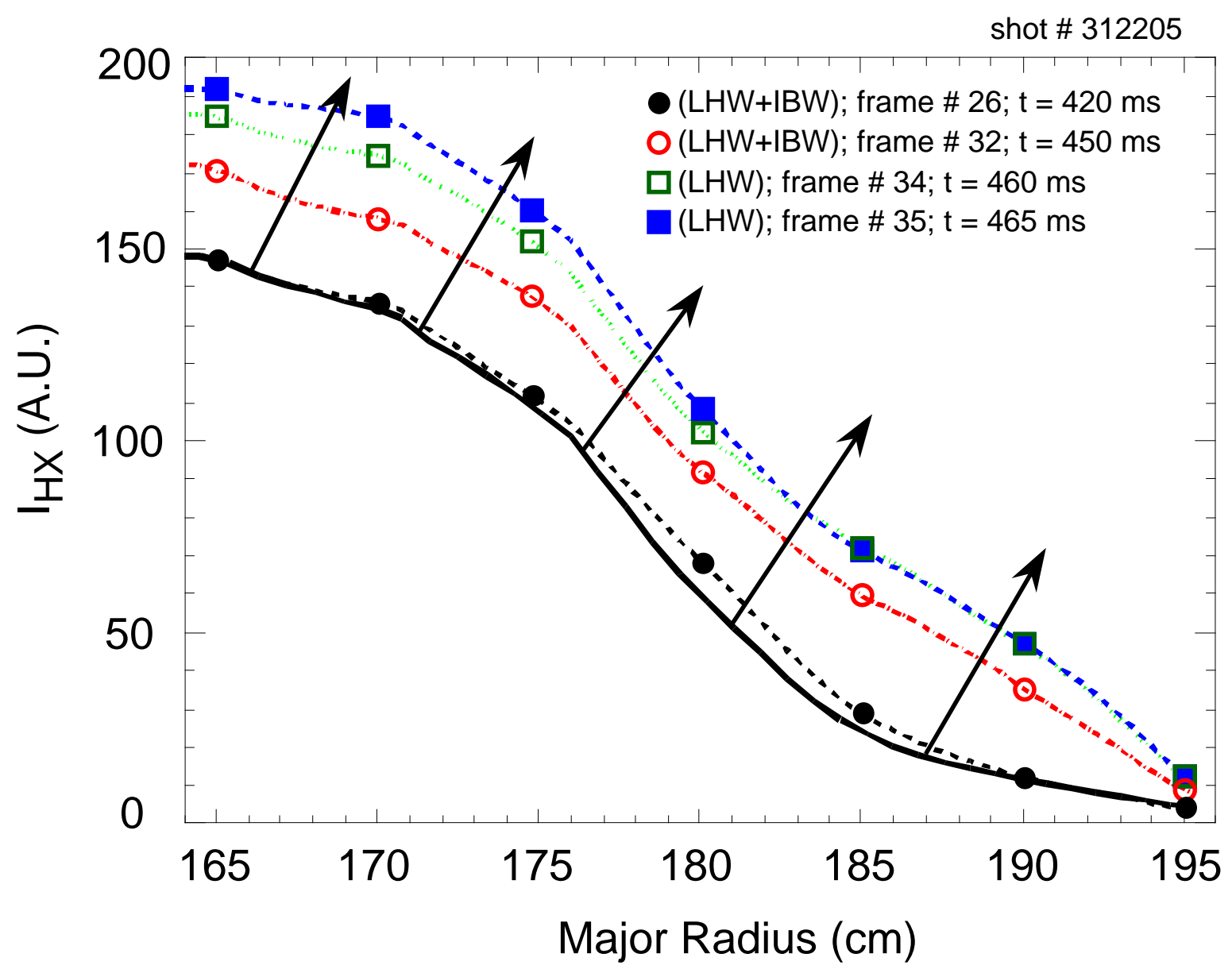

Figure 2

Vertical HXR intensity profiles at different time frames before and after the onset of IBW power. All the measurements are taken during the LHCD phase of discharge \# 312205. The continuous line is the LHW-only reference profile at $t=t L H W=400 \mathrm{~ms}$. The arrows indicate the evolution of the profiles $\operatorname{lHX}(\mathrm{t})$ in time.

The following table defines the different symbols:

$\begin{array}{llll}\text { filled circles } & t=420 \mathrm{~ms} & \text { frame \# 26 } & (\text { LHW + IBW) } \\ \text { empty circles } & t=450 \mathrm{~ms} & \text { frame \# 32 } & (\text { LHW + IBW }) \\ \text { empty squares } & t=460 \mathrm{~ms} & \text { frame \# 34 } & (\text { LHW }) \\ \text { filled squares } & t=465 \mathrm{~ms} & \text { frame \# 35 } & (\text { LHW })\end{array}$


shot \# 312205

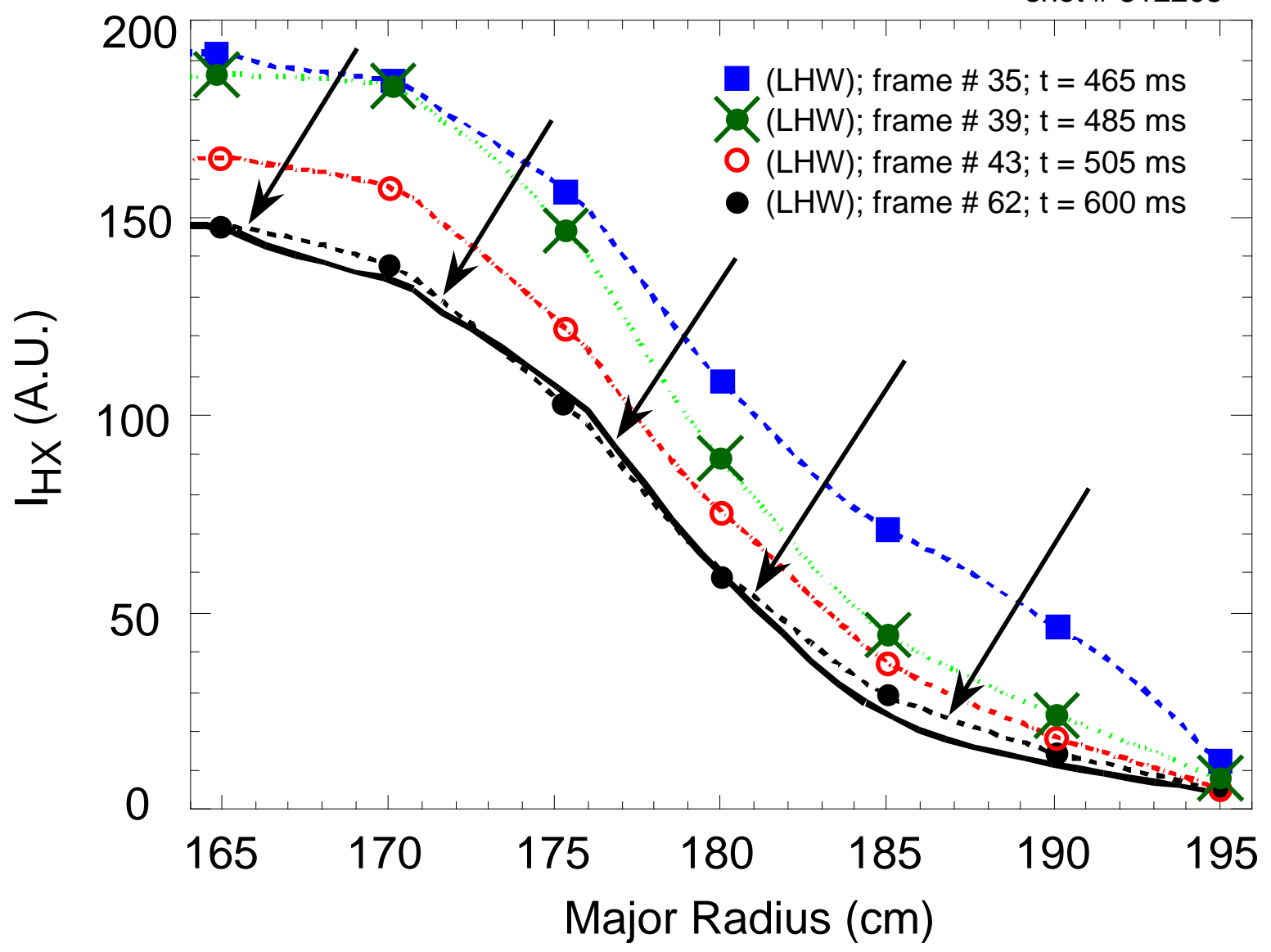

Figure 3

Same as in Fig. 2 but at different time frames during the relaxation period, after the IBW power has been turned off. The arrows indicate the evolution of the profiles $\operatorname{IHX}(\mathrm{t})$ in time.

The following table defines the different symbols:

$\begin{array}{llll}\text { filled squares } & t=465 \mathrm{~ms} & \text { frame \# 35 } & \text { (LHW) } \\ \text { filled circles over crosses } & t=485 \mathrm{~ms} & \text { frame \# 39 } & \text { (LHW) } \\ \text { empty circles } & t=505 \mathrm{~ms} & \text { frame \# 43 } & \text { (LHW) } \\ \text { filled circles } & t=600 \mathrm{~ms} & \text { frame \# 62 } & \text { (LHW) }\end{array}$




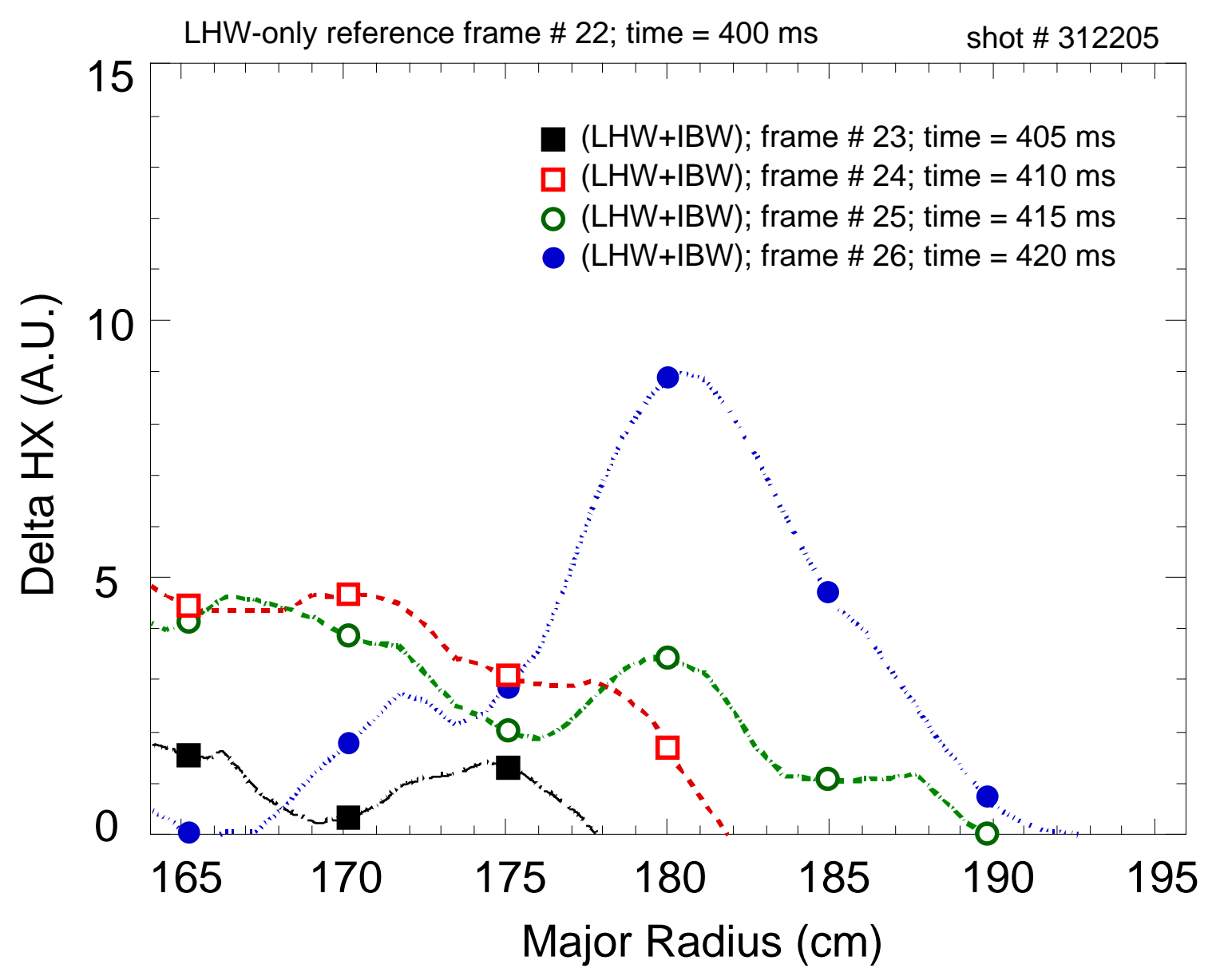

Figure 4

Spatial profiles of the variable $\Delta \mathrm{HX}(\mathrm{t})$ at several time frames after the onset of IBW power.

All the measurements are taken during the LHCD phase of discharge \# 312205.

The following table defines the different symbols:

$\begin{array}{llll}\text { filled squares } & t=405 \mathrm{~ms} & \text { frame \# 23 } & \text { (LHW + IBW) } \\ \text { empty squares } & t=410 \mathrm{~ms} & \text { frame \# 24 } & \text { (LHW + IBW) } \\ \text { empty circles } & t=415 \mathrm{~ms} & \text { frame \# 25 } & \text { (LHW + IBW) } \\ \text { filled circles } & t=420 \mathrm{~ms} & \text { frame \# 26 } & \text { (LHW + IBW) }\end{array}$




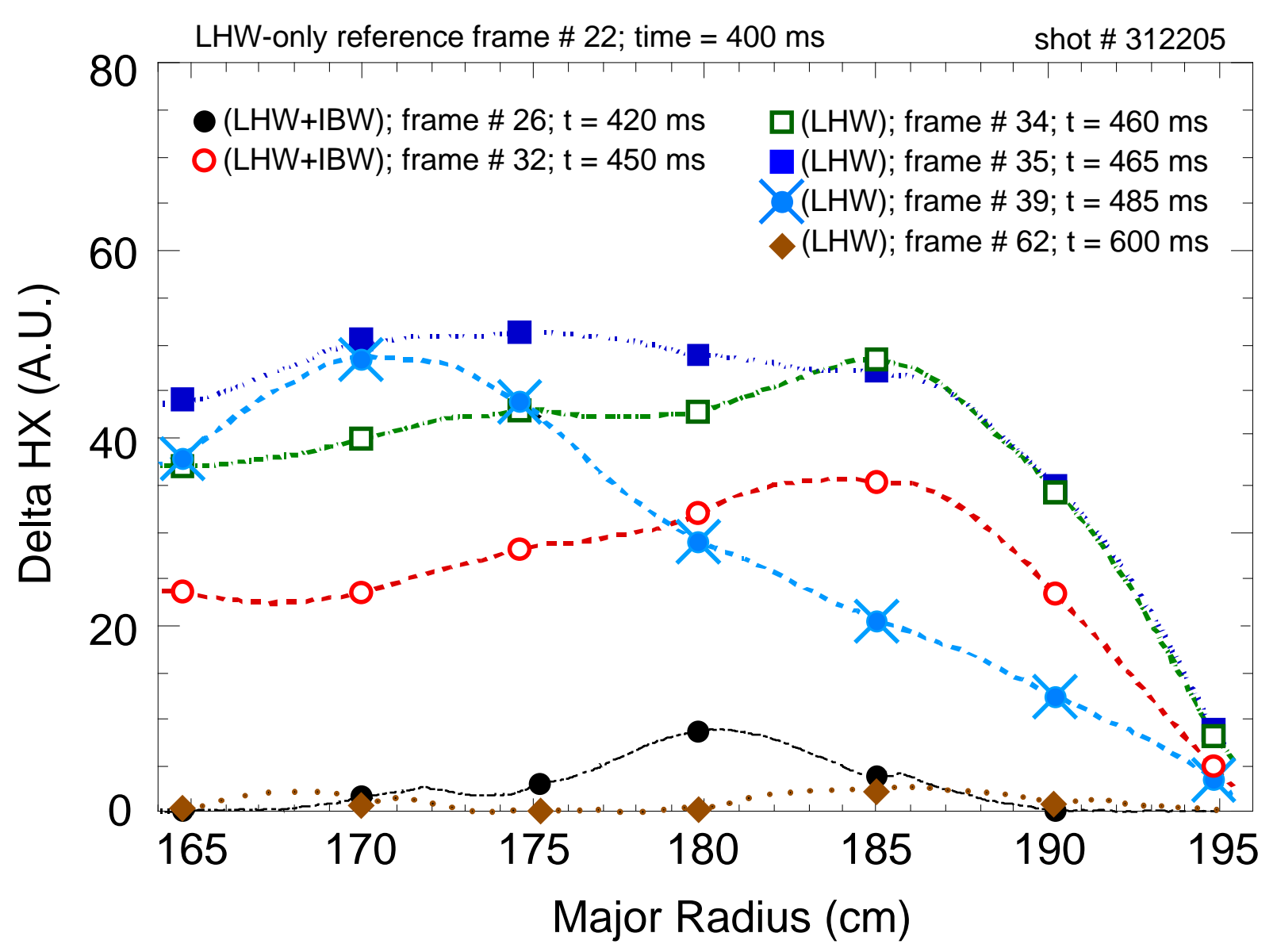

Figure 5

Same as in Fig. 4 but at different time frames before and after the IBW power is turned off.

The following table defines the different symbols:

$\begin{array}{llll}\text { filled circles } & t=420 \mathrm{~ms} & \text { frame \# 26 } & (\mathrm{LHW}+\mathrm{IBW}) \\ \text { empty circles } & \mathrm{t}=450 \mathrm{~ms} & \text { frame \# 32 } & (\mathrm{LHW}+\mathrm{IBW}) \\ \text { empty squares } & \mathrm{t}=460 \mathrm{~ms} & \text { frame \# 34 } & (\mathrm{LHW}) \\ \text { filled squares } & \mathrm{t}=465 \mathrm{~ms} & \text { frame \# 35 } & (\mathrm{LHW}) \\ \text { filled circles over crosses } & \mathrm{t}=485 \mathrm{~ms} & \text { frame \# 39 } & (\mathrm{LHW}) \\ \text { diamonds } & \mathrm{t}=600 \mathrm{~ms} & \text { frame \# 62 } & (\mathrm{LHW})\end{array}$




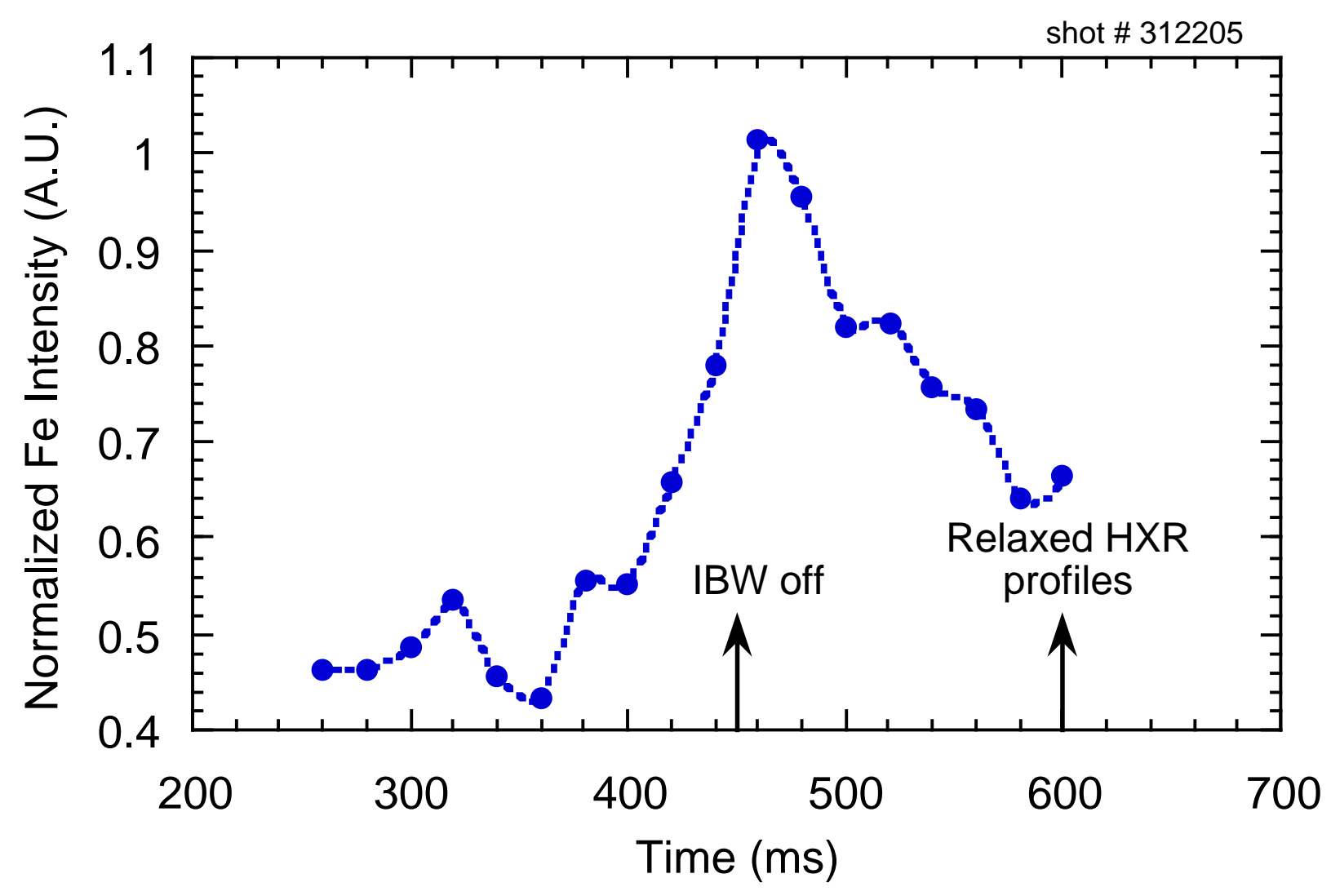

Figure 6

Time behavior of the centrally emitting $335 \AA$ line from Fe XVI normalized to the electron density. The emission is detected by the SPRED diagnostic on discharge \# 312205. 


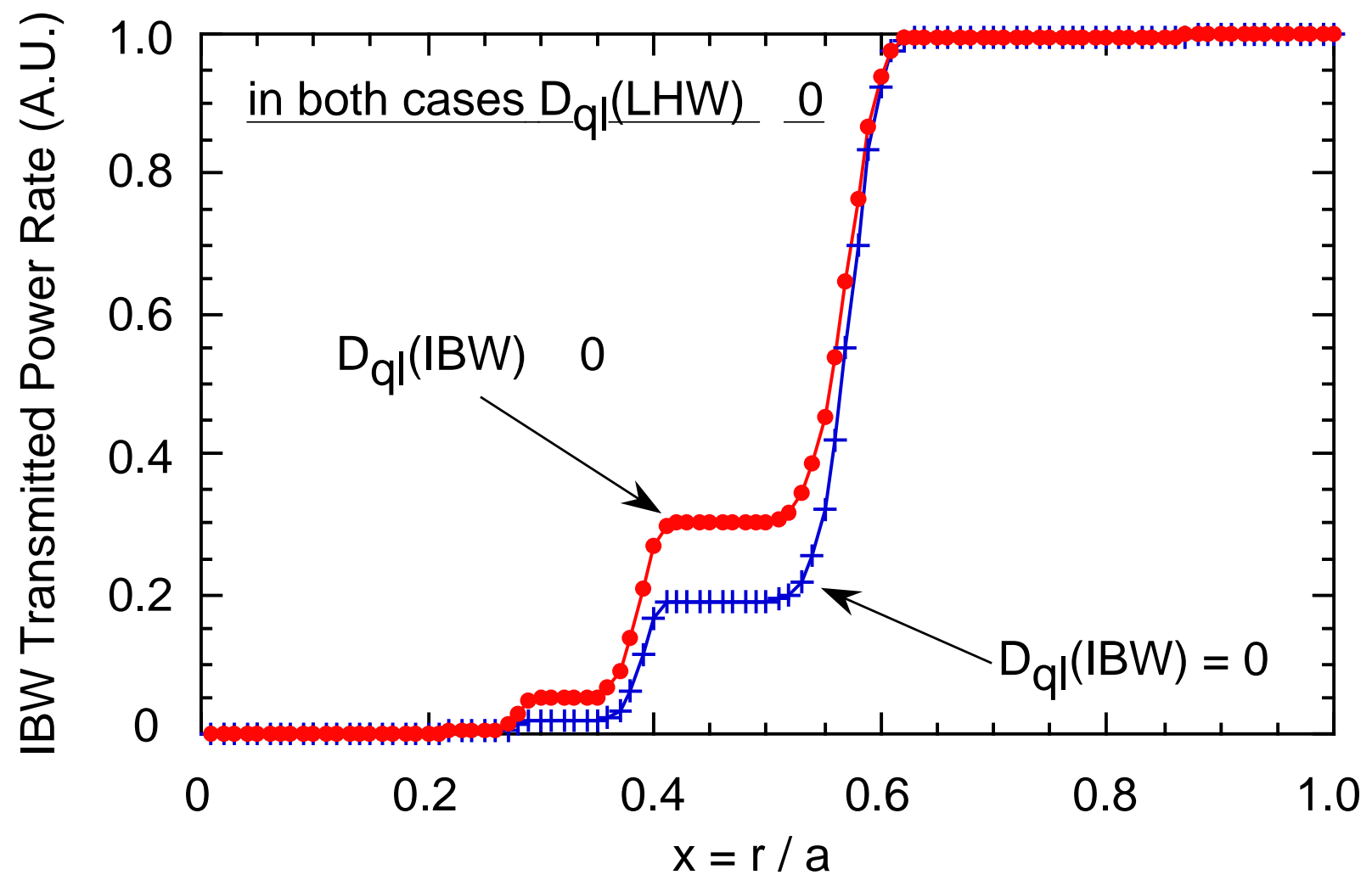

Figure 7

Comparison between IBW transmitted power rate radial profiles in a case with $\mathrm{DQL}(\mathrm{IBW}) \neq 0$ (filled circles) and a case with $\mathrm{DQL}(\mathrm{IBW})=0$ (crosses). For both cases $\mathrm{DQL}(\mathrm{LHW}) \neq 0$. Parameters of the plasma and waves used in this simulation are shown in Tab. 1,2 respectively. 


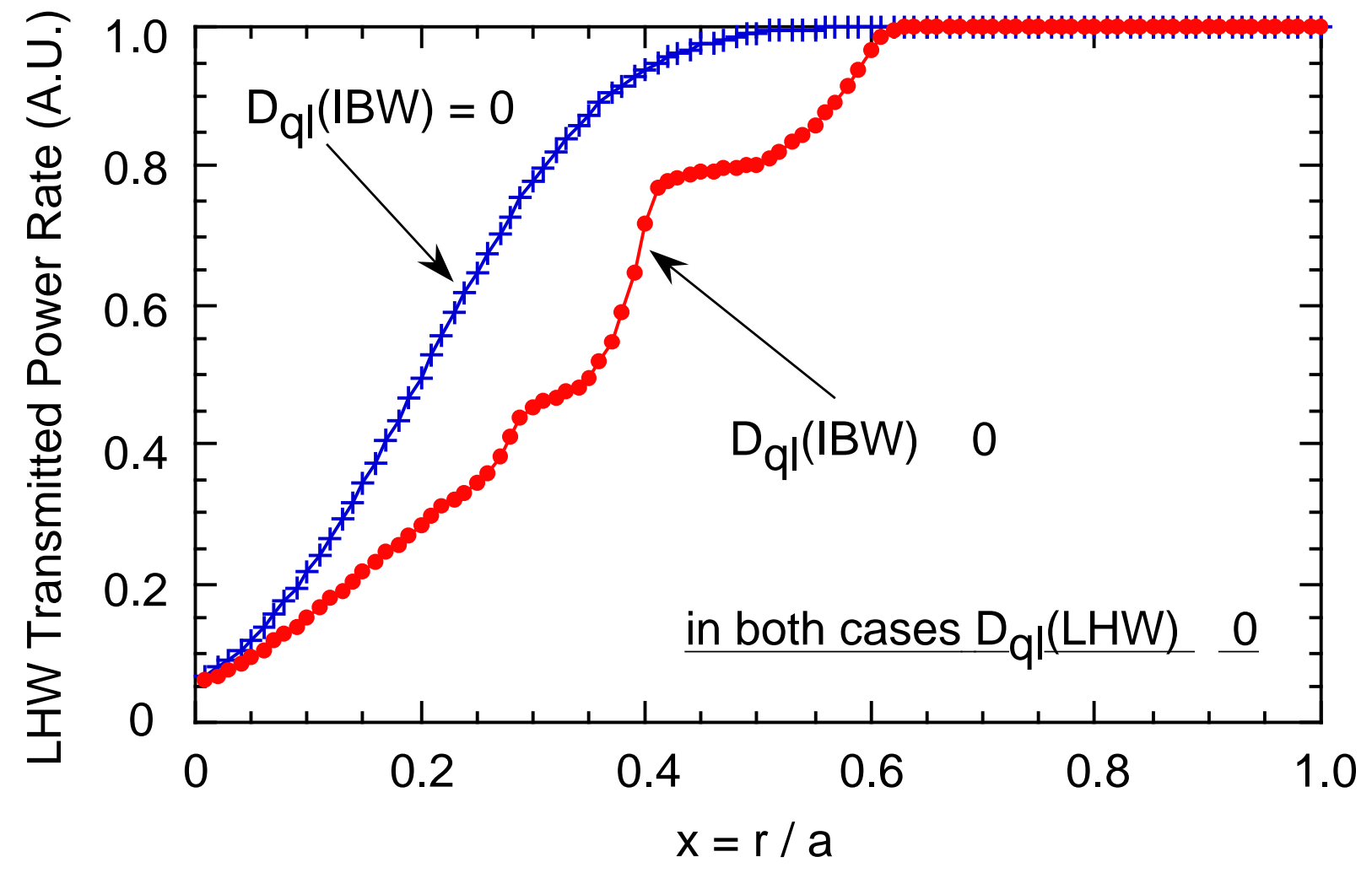

Figure 8

Comparison between LHW transmitted power rate radial profiles in the same cases shown in Fig. 7. 


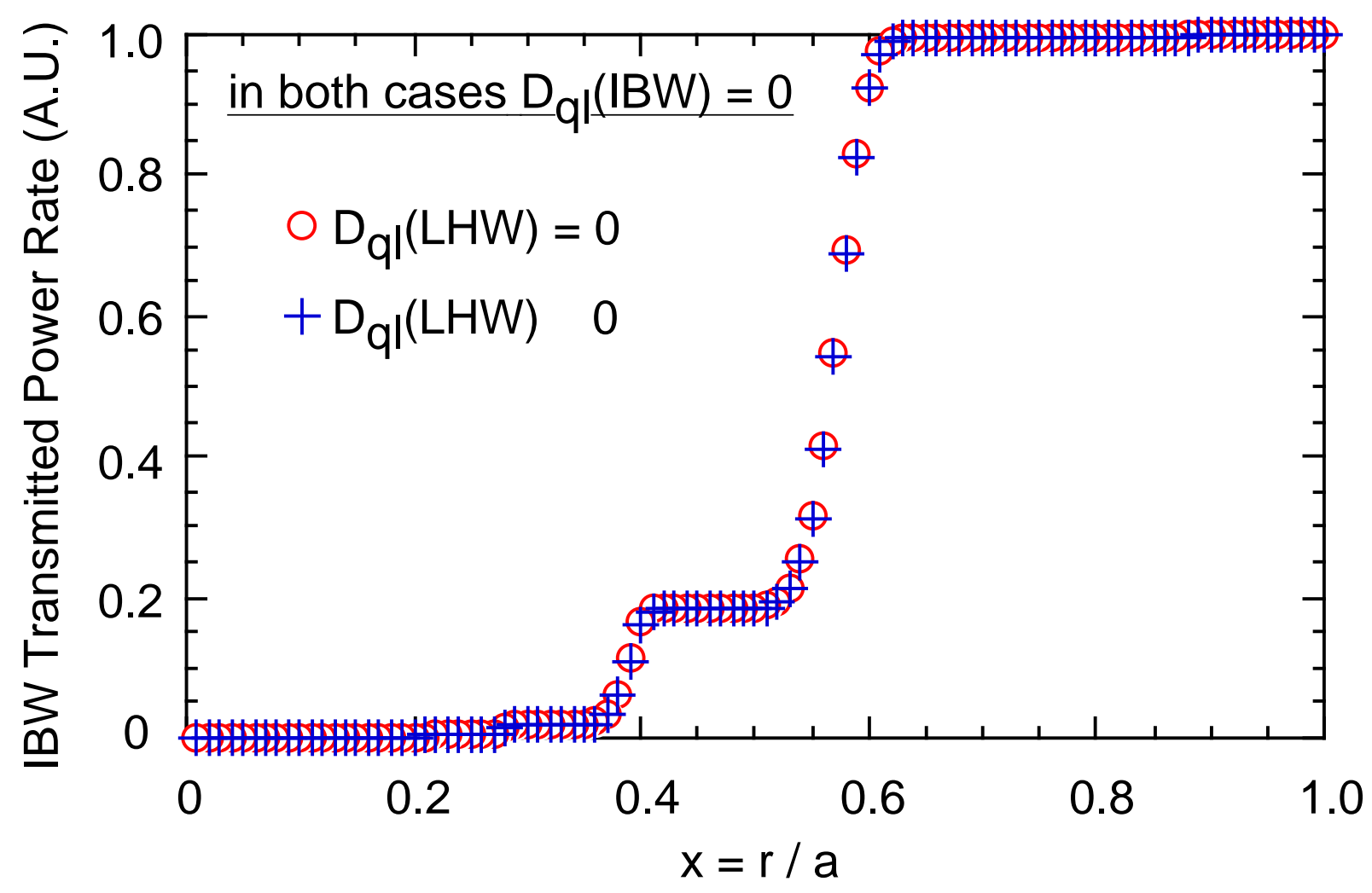

Figure 9

Comparison between IBW transmitted power rate radial profiles in a case with $\mathrm{DQL}(\mathrm{LHW})=0$ (empty circles) and a case with $\mathrm{DQL}(\mathrm{LHW}) \neq 0$ (crosses). For both cases $\mathrm{DQL}(\mathrm{IBW})=0$. Parameters of the plasma and waves used in this simulation are the same as in Fig. 7. 


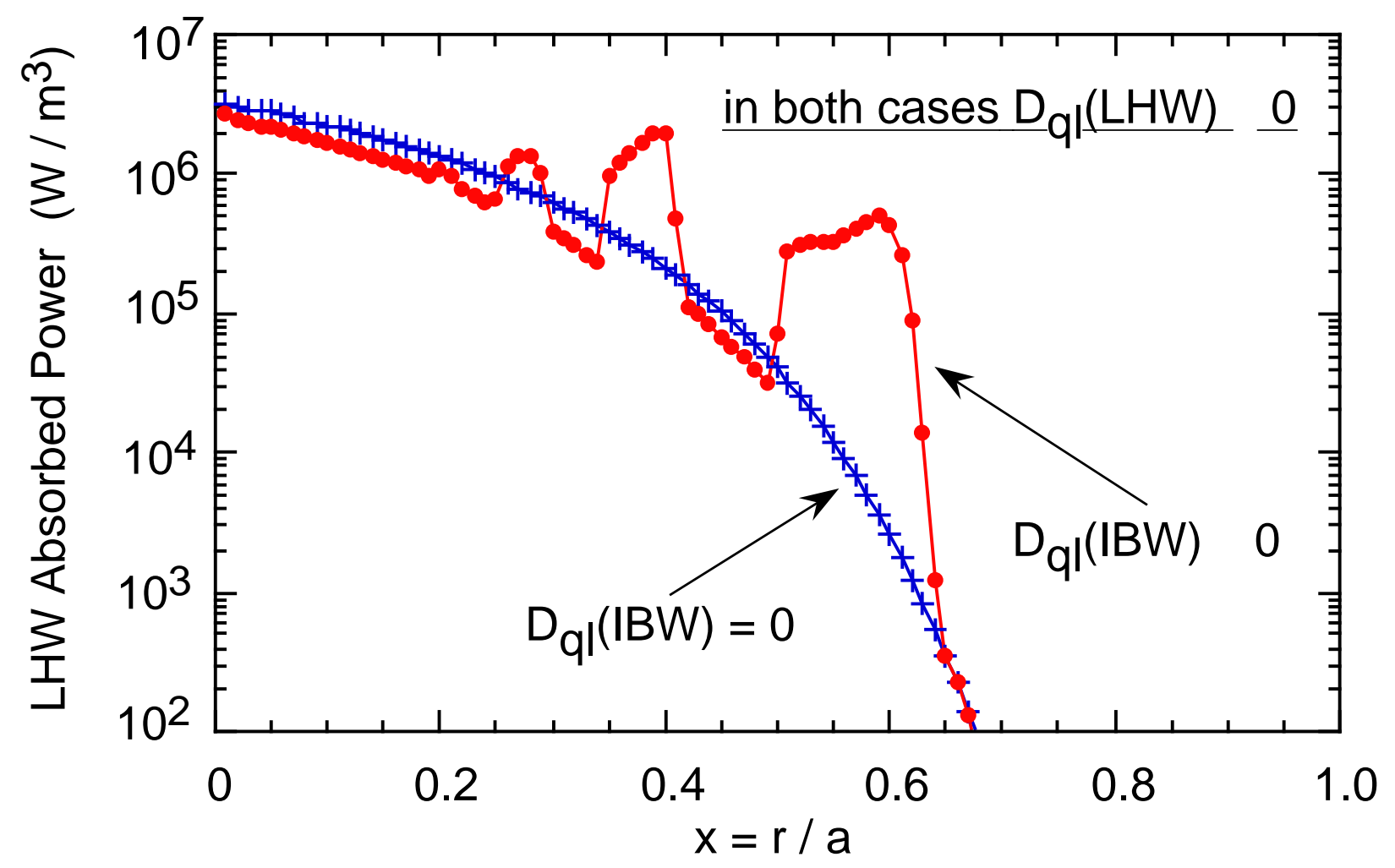

Figure 10

Comparison between the total (LHW + IBW) absorbed power profiles in the same cases shown in Fig. 7. The vertical axis is a logarithmic scale. 


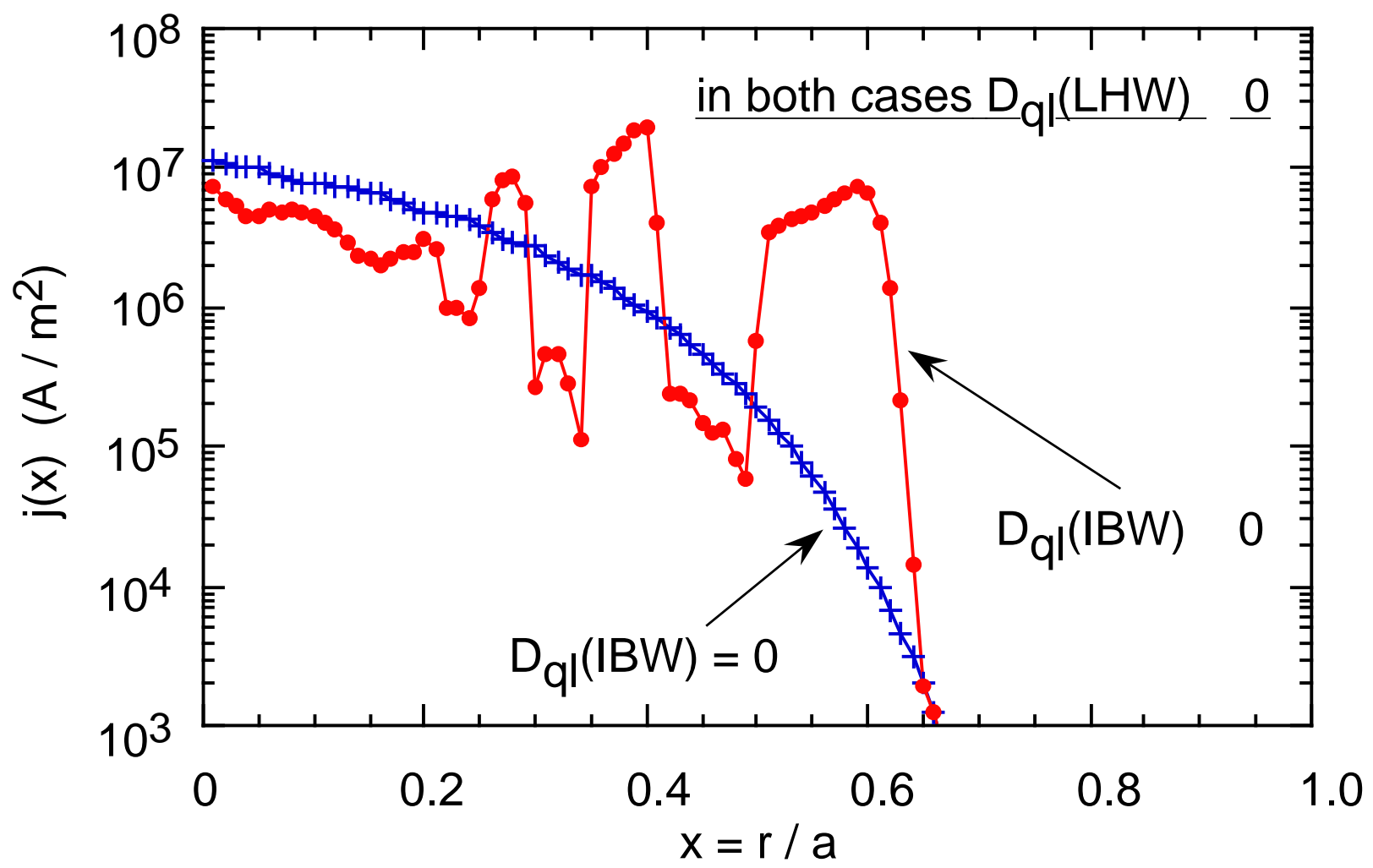

Figure 11

Comparison between the calculated non inductive current density profiles in the same cases shown in Fig. 7. The vertical axis is a logarithmic scale. 


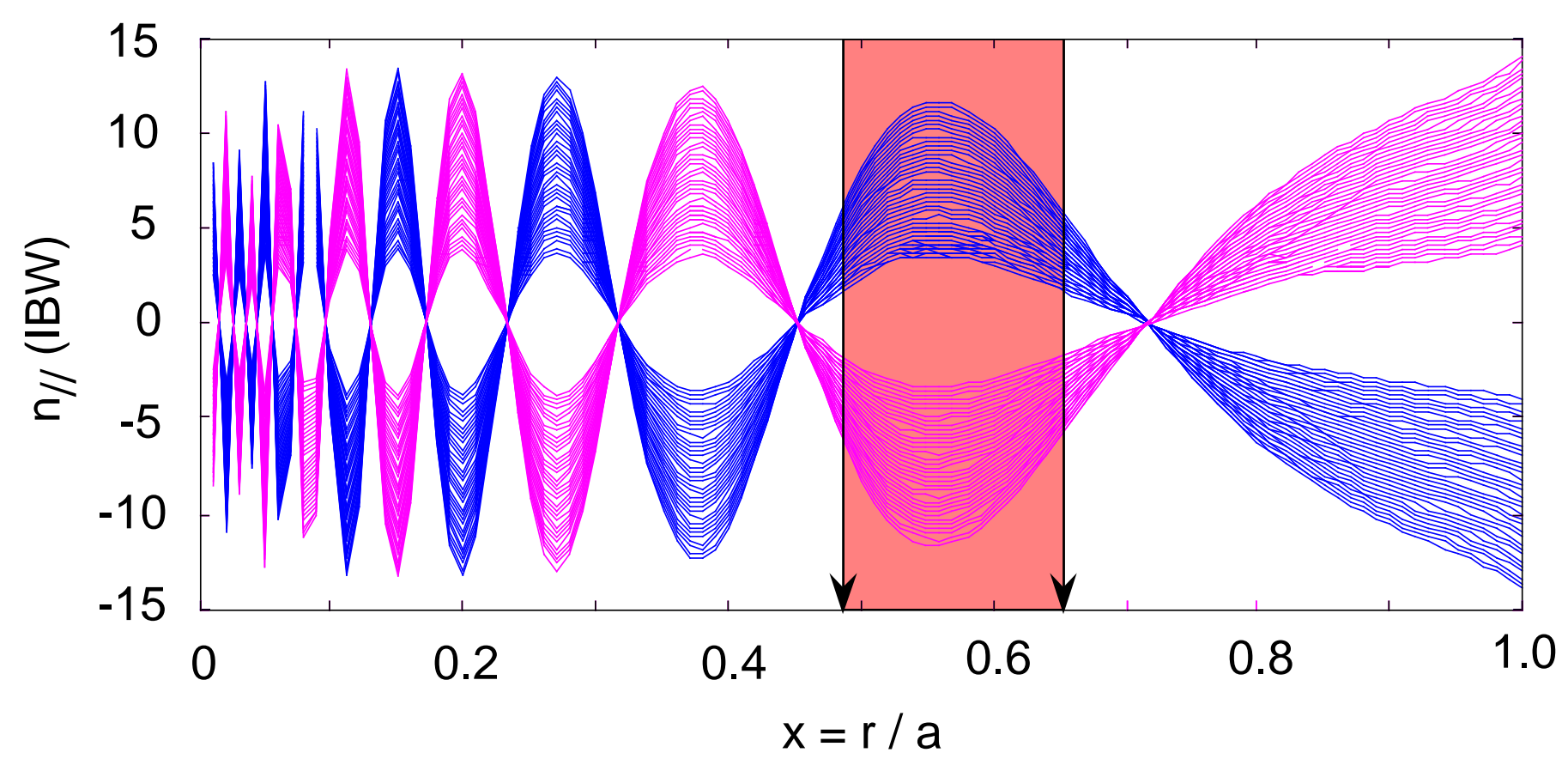

Figure 12

IBW $n_{\|}$radial profile from the solution of the WKBJ ray-tracing equations. The shaded region has been defined as "region of strong synergistic interaction" around the first IBW $n_{\|}$maximum. Parameters of the plasma and waves used in this simulation are the same as in Fig. 7 . 